\title{
Handeln statt wegsehen!
}

\section{Das Diakonische Werk Berlin-Brandenburg-schlesische Oberlausitz engagiert sich gegen Rechts}

Christiane Lehmacher-Dubberke

Auch soziale Organisationen sind mit rechtsextremen Einstellungen von Mitarbeitenden und Nutzern konfrontiert. Das Diakonische Werk Berlin-Brandenburgschlesische Oberlausitz will in einer Initiative mit anderen Organisationen dagegen vorgehen.

»Manager und Mitarbeiter können durch ein Klima der Toleranz in den Unternehmen dafür sorgen, dass rechtsextremistisches Gedankengut keine Chance hat. Das Betriebsklima wirkt in die Gesellschaft zurück, denn Firmen sind wichtige Orte der Meinungsbildung. «Das sagte Staatssekretär Clemens Appel, Chef der Staatskanzlei des Landes Brandenburg, tremismus. Er stellt Hintergrundwissen zur Verfügung und weist auf weiterführende Beratungs- und Unterstützungsangebote hin. Alle Texte und Materialien können auf einer Internetseite heruntergeladen werden (www.handeln-statt-wegsehen.de).

Auch das Diakonische Werk BerlinBrandenburg-schlesische Oberlausitz hat sich dieser Initiative angeschlossen. Diakoniedirektorin Susanne Kahl-Passoth dazu: "Wir dürfen nicht die Augen davor verschließen, dass Rechtsextremismus, Antisemitismus und Rassismus ein Problem in unserer Gesellschaft sind. Welche Strukturen sich rechtsextreme Gruppierungen in der Zwischenzeit geschaffen haben, mit welchem Know-how sie z. B.

\section{$»$ Rechtsextreme lassen sich beispielsweise in Elternbeiräte wählen «}

vor Vertretern des Handels und Gewerkschaftsmitgliedern in der Gedenkstätte Sachsenhausen. Der Handelsverband Berlin-Brandenburg (HBB), ver.di Berlin, der DGB Bezirk Berlin-Brandenburg und die "Mobile Beratung gegen Rechtsextremismus « hatten hier am 14. Oktober 2008 zum Auftakt der Initiative »Handeln statt wegsehen! « eingeladen.

Das Ziel der gemeinsamen Initiative besteht darin, in den Betrieben und Berufsschulen die Auseinandersetzung mit der Problematik des Rechtsextremismus zu fördern. Durch die Bereitstellung von Informationsmaterialien, die Organisation von Workshops, die Vermittlung von Referenten und weiterer Angebote sollen Ausbilder und Lehrer sowie die Geschäftsleitungen der einzelnen Unternehmen entsprechend unterstützt werden. Betriebe und Schulen, die sich der Initiative anschließen möchten, erhalten vom Handelsverband Berlin-Brandenburg einen "Infokoffer « als Starthilfe bei der Auseinandersetzung mit Rassismus, Antisemitismus und Rechtsex-
Mitglieder werben, wird bedauerlicherweise noch stark unterschätzt. In den Köpfen vieler Menschen sind Rechtsextreme agitierende Massen, die Parolen brüllen - aber die Realität ist anders. Menschen aus der gesellschaftlichen Mitte werben scheinbar harmlos für >bürgerliche Rechte`, gehen z. B. verstärkt in Gremien und werben an Schulen. «

Schon seit längerem beobachtet das Diakonische Werk diese Problematik, die vielfältige Trägerlandschaft diakonischer Einrichtungen verschafft dabei einen durchaus repräsentativen Eindruck des Einflusses rechtsradikaler Gruppierungen in den verschiedenen Bevölkerungsschichten der Länder Berlin und Brandenburg. Ein Beispiel für die gezielte Vorgehensweise ist, dass sich zunehmend rechtsextreme Eltern in Elternbeiräte etc. wählen lassen, um so Einfluss und neue Mitglieder zu gewinnen. In dieser Situation fühlen sich die Erzieherinnen und Erzieher häufig hilflos, weil es ihnen an einem geeigneten Argumentationsinstru- 
mentarium fehlt, rechtsradikalem Gedankengut effektiv zu begegnen und bereits so von Anfang an die Stirn zu bieten. Den Strategien rechtsradikaler Vereinigungen oder rechtsradikalem Gedankengut muss frühzeitig mit ebenfalls intelligenten Strategien konsequent begegnet werden.

Für die Mitgliedseinrichtungen des Diakonischen Werkes wird es zu diesem Thema eine Fachveranstaltung geben. Das Angebot wendet sich in einem ersten Schritt an die Träger mit einem pädagogischen Auftrag, seien es Kitas, Schulen oder Berufsschulen. Die pädagogischen Mitarbeiterinnen und Mitarbeiter sollen sensibilisiert und in einem nächsten Schritt durch ergänzende Angebote beraten und unterstützt werden. Susanne Kahl-Passoth betont: »Die Fachveranstaltung ist wahrscheinlich der Auftakt zu einem Themenschwerpunkt, der uns durch die nächsten Jahre begleiten wird. Das ist unsere Befürchtung, aber wir werden auch alles dafür tun, um es den rechten Kräften in unserem Land schwer zu machen, Fuß zu fassen! Wir handeln!«

\section{Rechtsextremismus im Betrieb}

Verbreitet ein Mitarbeiter, ob Arbeitnehmer oder Auszubildender, im Betrieb fremdenfeindliche, rassistische oder nazistische Parolen, belästigt oder bedroht er ausländische Kollegen oder betätigt er sich in anderer die Menschenwürde missachtender Weise, dann begeht er möglicherweise nicht nur eine Straftat, sondern verletzt elementare Pflichten aus dem Arbeitsverhältnis. In diesen Fällen kann der Arbeitgeber dem Betreffenden außerordentlich (fristlos) oder ordentlich (unter Einhaltung der Kündigungsfrist) - gegebenenfalls nach vorheriger Abmahnung - kündigen, wenn durch dieses Verhalten der Betriebsfrieden oder der betriebliche Arbeitsablauf gestört werden.

Nach der Rechtsprechung des Bundesarbeitsgerichts ist die vorherige Abmahnung des Arbeitnehmers durch den Arbeitgeber nicht erforderlich, wenn es sich um eine schwere Pflichtverletzung handelt, deren Rechtswidrigkeit für den Arbeitnehmer ohne weiteres erkennbar und bei der eine Hinnahme des Verhaltens offensichtlich ausgeschlossen ist (Urteil vom 1. Juli 1999, Der Betrieb 1999. S. 2216).

Ein Arbeitgeber muss nicht tatenlos zusehen und erst reagieren, wenn im Betrieb etwas passiert ist. Er kann z. B. in Abstimmung mit dem Betriebsrat durch Rundschreiben, Veröffentlichung in der Betriebszeitung oder durch Übergabe eines Informationsblattes eindeutig klarstellen, dass Ausländerfeindlichkeit und Rassismus im Betrieb nicht geduldet werden und zur Entlassung führen können.

Quelle: Website des Netzwerks Sachsen gegen Rechtsextremismus, Gewalt und Fremdenfeindlichkeit e. V.

Internet http://www.netzwerk-sachsen.de/html/betrieb.html

\title{
Rassismus, Antisemitismus und Rechtsextremismus haben in unserem Betrieb keinen Platz!
}

\section{Wir tolerieren keine diskriminierenden Äußerungen oder körperlichen Angriffe, zum Beispiel wegen Hautfarbe, Religion, Nationalität oder sexueller Orientierung!}

\author{
Wir stehen für Gleichbehandlung \\ und Achtung der Menschenwürde ein!
}

Der »Antidiskriminierungskodex « aus einem Infokoffer der Initiative » Handeln statt wegsehen! « ist als Aushang in Betrieben und Organisationen gedacht. Weiteres Material bietet die Website der Intitative (http://www.handeln-statt-wegsehen.de). 